\title{
Curva de Crescimento do Diâmetro Biparietal e da Circunferência Cefálica na Gestação Gemelar
}

Growth Curve of the Biparietal Diameter and Head Circumference in Twin Gestation

Mariza Marie Fujita ${ }^{1}$, Maria Okumura ${ }^{1}$, Julio da Motta Singer ${ }^{2}$, Dalton Francisco de Andrade ${ }^{2}$, Marcelo Zugaib ${ }^{1}$

\begin{abstract}
RESUMO
Objetivo: determinar curvas e tabelas de crescimento do diâmetro biparietal médio e da circunferência cefálica média em relação à idade gestacional de gestações gemelares e analisar eventuais diferenças com as existentes para fetos únicos e gêmeos.

Métodos: por meio de exames ultra-sonográficos realizados a cada duas ou três semanas por um único observador em 34 pacientes com gestação gemelar sem patologias feto-maternas foi possivel estabelecer curvas e tabelas do diâmetro biparietal médio e da circunferência cefálica média em relação a idade gestacional. Os valores destes parâmetros biométricos foram comparados aos descritos pela literatura para fetos únicos e gêmeos.

Resultados: foram obtidas curvas e tabelas do diâmetro biparietal e da circunferência cefálica em relação a idade gestacional. O crescimento do diâmetro biparietal e da circunferência cefálica se revelou menor no terceiro trimestre em relação aos observados por Hadlock et al. ${ }^{15}$ para gestação única. A diferença entre os valores médios foi $6 \mathrm{~mm}$ (diâmetro biparietal) e 2,0 cm (circunferência cefálica) na 39a semana de gestação. Foram observadas também algumas diferenças em relação a outros estudos de gestações gemelares.

Conclusão: este estudo sugeriu que as curvas e tabelas de crescimento do diâmetro biparietal médio e da circunferência cefálica média de gestações únicas não são apropriadas para avaliação de gestações gemelares da população analisada principalmente no terceiro trimestre.
\end{abstract}

PALAVRAS-CHAVE: Gemelaridade. Ultra-sonografia fetal. Crescimento fetal. Diâmetro biparietal.

\section{Introdução}

Há muitos estudos estabelecendo padrões de desenvolvimento normal de vários parâmetros biométricos ultra-sonográficos durante a gestação. Estes padrões são utilizados para avaliar o crescimento fetal tanto de gestações únicas quanto de

Hospital das Clínicas da Faculdade de Medicina da Universidade de São Paulo (HCFMUSP)

${ }^{1}$ Departamento de Obstetrícia e Ginecologia do Hospital das Clínicas da Faculdade de Medicina da Universidade de São Paulo

2 Departamento de Estatística do Instituto de Matemática e Estatistíca da Universidade de São Paulo

Correspondência: Mariza Marie Fujita

Rua Antônio Loureiro, 594 - Apto 31C - Vila Santa Catarina 04376-110 - São Paulo - SP gestações gemelares. Porém, há dúvidas se as curvas de crescimento normal obtidas por meio da ultra-sonografia para gestação única podem ser utilizadas para o acompanhamento de gestações múltiplas ${ }^{1,2}$.

$\mathrm{Na}$ literatura, verifica-se que as curvas e tabelas dos parâmetros biométricos ultrasonográficos de gestações gemelares foram elaboradas sem o devido rigor na seleção das pacientes e sem o cuidado necessário em relação aos aspectos técnicos e na determinação da idade gestacional.

Em vista disso, este estudo se propõe a determinar curvas de crescimento do diâmetro biparietal médio e da circunferência cefálica média de gestações gemelares sem patologias feto- 
maternas, por meio de estudo prospectivo e longitudinal efetuado por um único observador. Com tais cuidados, pretende-se minimizar a margem de erros técnicos e a influência de outros fatores que afetam o crescimento fetal, para que as curvas obtidas reflitam mais fielmente o crescimento intra-uterino peculiar de gestações gemelares. Este estudo tem como objetivo ainda, analisar eventuais diferenças entre as curvas obtidas na população em estudo e as curvas existentes para fetos únicos e gêmeos.

\section{Pacientes e Métodos}

Foram realizados exames ultra-sonográficos em 98 pacientes com gestação gemelar no Ambulatório de Pré-natal da Clínica Obstétrica do Hospital das Clínicas da Faculdade de Medicina da Universidade de São Paulo (HCFMUSP), no período de junho de 1994 a maio de 1997, tendo sido selecionadas 34 pacientes, conforme os seguintes critérios de inclusão: (1) ausência de qualquer patologia materna intercorrente durante a gestação, (2) ausência de malformação ou óbito fetal, (3) primeira ultra-sonografia obstétrica realizada até a 24a semana de gestação, (4) ausência de discordância de peso entre os gêmeos, sendo a mesma definida como diferença igual ou maior que $20 \%$ do peso do maior gêmeo ao nascimento e (5) gestações gemelares duplas.

Foram realizados exames ultra-sonográficos fetais a cada duas ou três semanas sempre por um único observador. Cada medida foi efetuada pelo menos três vezes, empregando para analise a média das mesmas. Não se consideraram medidas provenientes de imagens ultra-sonográficas insatisfatórias.

A idade gestacional (IG) foi estabelecida a partir da data do primeiro dia da última menstruação desde que se apresentasse compativel com a primeira ultra-sonografia, ou seja, estivesse dentro do intervalo de variabilidade descrito para aquela idade gestacional ${ }^{3}$. Quando a data do primeiro dia da última menstruação era desconhecida, a idade gestacional considerada foi a obtida pela primeira ultra-sonografia com a medida do comprimento cabeça-nádega ${ }^{4}$ ou pela média aritmética da idade gestacional estimada pelo diâmetro biparietal $(\mathrm{DBP})^{5}$, circunferência cefálica $(\mathrm{CC})^{6}$, circunferência abdominal $(\mathrm{CA})^{7}$ e comprimento do fêmur $(F)^{8}$, conforme o preconizado por Hadlock et al. ${ }^{3}$. A idade gestacional foi relatada em semanas completas, como recomendado pela World Health Organization $^{9}$, ou seja, até um dia antes de que se complete a semana seguinte. Assim, 30 sema- nas incluem idades gestacionais de 210 até 216 dias.

A primeira ultra-sonografia obstétrica foi realizada até a $24^{\text {a }}$ semana das quais $26,5 \%$ até a $12^{\text {a }}$ semana, $50 \%$ entre a $13^{\mathrm{a}}$ e $20^{\mathrm{a}}$ semana e $23,5 \%$ entre a $21^{\text {a }}$ e $24^{\text {a }}$ semana. A data do primeiro dia da última menstruação foi referida por 25 pacientes e não-conhecida por 9 , sendo que, nestas, a primeira ultra-sonografia foi realizada até a $12^{\text {a }}$ semana em 1 paciente, entre a $13^{\text {a }}$ e a $20^{a}$ semana em 7 pacientes e entre a $21^{\underline{a}}$ e a $24^{a}$ semana em 1 paciente.

Os exames foram realizados em aparelho de ultra-sonografia da marca Toshiba, modelo Capasee SSA-220A, de varredura convexa eletrônica, com freqüência de emissão de $3,75 \mathrm{MHz}$, visibilização modo B e capacidade de magnificação da imagem. As técnicas para obtenção de imagem e medidas foram as descritas na literatura ${ }^{10}$.

Para medir o diâmetro biparietal e a circunferência cefálica, examinou-se a cabeça fetal em secção axial transversa, visualizando-se a foice, o cavo do septo pelúcido, o núcleo do tálamo e o plexo coróide no átrio dos ventrículos laterais. Foram medidos o diâmetro biparietal (D1) da superficie externa da tábua craniana próxima ao transdutor à superficie interna da tábua craniana distal e o diâmetro occipitofrontal (D2) da margem externa à margem externa da tábua craniana oposta por meio de medidores eletrônicos. A circunferência cefálica foi calculada por meio da fórmula para circunferência de uma elipse (D1 + D2) x 1,57 ${ }^{11}$.

Os exames ultra-sonográficos também incluiram determinação da apresentação fetal, estimativa do volume de líquido amniótico e localização placentária, além do estudo anatômico completo dos fetos.

A idade das 34 pacientes variou entre $17 \mathrm{e}$ 40 anos (média de $29 \pm 6$ anos) e em relação à cor, $50 \%$ eram brancas e $50 \%$ não-brancas. Quanto ao número de gestações, $8(23,5 \%)$ eram primigestas, $11(32,4 \%)$ secundigestas e $15(44,1 \%)$ haviam tido três ou mais gestações. Segundo a paridade a distribuição indicou $35,3 \%$ de nulíparas, $26,5 \%$ de primíparas e $38,2 \%$ de multíparas.

A idade gestacional na ocasião do parto situou-se entre 34 e 39 semanas (média de $37 \pm 1$ semanas). $O$ parto de cinco pacientes não ocorreu no HCFMUSP, impossibilitando a obtenção de alguns dados, como o índice de Apgar e o índice de Capurro.

Quanto ao tipo de gestação gemelar, 22 pares eram dizigóticos, 7 pares monozigóticos e de 5 não se obteve essa informação. O tipo de gestação, se monozigótica ou dizigótica, foi determinado pelo sexo, pelo exame anatomopatológico da placenta, pelo tipo sangüíneo e pelo fenótipo dos gêmeos após 
seis meses de vida. A análise do tipo de gestação gemelar quando conhecida e o exame anatomopatológico das placentas revelaram placentas dicoriônicas em 23 pares, monocoriônicas em 3 pares e não se obteve tal informação de 8 pares.

Em relação ao sexo, 40 recém-nascidos eram do sexo feminino $(58,8 \%)$ e 28 do masculino $(41,2 \%)$, sendo a distribuição dos pares a seguinte: $44,1 \%$ feminino-feminino, $26,5 \%$ masculinomasculino e $29,4 \%$ feminino-masculino.

$\mathrm{O}$ peso ao nascimento variou entre $1.780 \mathrm{e}$ $3.210 \mathrm{~g}$ (média $=2.505 \pm 326 \mathrm{~g}$ ). A diferença relativa do peso ao nascimento entre o par (feto menor em relação ao maior) foi de 0 a $18 \%$ (média $=8,4 \pm$ 4,6\%).

O índice de Apgar médio de 10 minuto foi 8 com desvio padrão de 2 , o de 5o minuto foi $9 \mathrm{com}$ desvio padrão de 1 e o de $10^{\circ}$ minuto foi $9 \mathrm{com}$ desvio padrão de 1 .

Em 58 recém-nascidos, a idade gestacional pelo método de Capurro et al. ${ }^{12}$ foi avaliada pela equipe de pediatras do Serviço de Pediatria Neonatal anexo à Maternidade do HCFMUSP. Um modelo de regressão linear foi utilizado para se relacionar a idade gestacional determinada conforme o método adotado e aquela estimada pelo método de Capurro et al. ${ }^{12}$, sugerindo que são diretamente proporcionais, com coeficiente de proporcionalidade igual a $1,00 \pm 0,01$.

Foram utilizados modelos de efeitos aleatórios para a análise dos $\operatorname{dados}^{13}$, pois permitem o ajuste das curvas polinomiais sob diferentes estruturas de dependência entre as observações e permitem modelar variabilidades heterogêneas ao longo do período gestacional. Para cada um dos parâmetros biométricos estudados, oito modelos polinomiais foram ajustados por meio do método de máxima verossimilhança restrita ${ }^{13}$. A seleção do modelo mais adequado baseou-se nos critérios de Akaike e Schwarz e na análise dos resíduos ${ }^{14}$.

Este estudo foi aprovado previamente pela Comissão de Ética para Análise de Projetos de Pesquisa do HCFMUSP e, de acordo com as normas estabelecidas, as pacientes assinaram o termo de consentimento para participar deste trabalho.

\section{Resultados}

Foram realizados 518 exames ultrasonográficos em 68 fetos gemelares no período gestacional compreendido entre a $12^{\text {a }}$ e $39^{\text {a }}$ semana.

O número de fetos, as médias e os desvios padrão das medidas dos parâmetros biométricos observados em cada idade gestacional constam da Tabela 1.

\section{Diâmetro biparietal}

Foram obtidos os coeficientes e os respectivos erros padrão correspondentes ao modelo com melhor ajuste. Esse modelo pode ser escrito como: DBP médio $=-1,92728+0,29834 \mathrm{IG}+0,00440 \mathrm{IG}^{2}-$ $0,00013 \mathrm{IG}^{3}$

A Tabela 2 apresenta os valores médios estimados e os respectivos intervalos de confiança $(95 \%)$ do diâmetro biparietal médio para idades gestacionais entre 12 e 39 semanas.

A Figura 1 mostra o diâmetro biparietal médio estimado com respectivo intervalo de confiança (95\%) entre 12 e 39 semanas de gestação do presente estudo e o de fetos únicos obtidos por Hadlock et al. ${ }^{15}$.

O diâmetro biparietal médio estimado neste trabalho foi equivalente ao de Hadlock et al. ${ }^{15}$ entre a $12^{\text {a }}$ e $16^{\text {a }}$ semana e a $26^{\underline{a}}$ e $32^{\text {a }}$ semana de gestação, sendo discretamente maior entre a $17^{\text {a }}$ e 25를 semana e menor após a $32^{\text {a }}$ semana, ou seja, o limite superior do intervalo de confiança dos valores do diâmetro biparietal dos gêmeos encontrouse abaixo daqueles descritos por Hadlock et al. ${ }^{15}$ (Figura 1). A diferença entre os valores médios foi $6 \mathrm{~mm}$ na 39a semana de gestação.

Os valores do diâmetro biparietal médio de fetos gêmeos relatados por Gottlicher et al. ${ }^{16}$, Grumbach et al. ${ }^{1}$ e Reece et al. ${ }^{2}$ foram comparados com os deste estudo na Figura 2. Constatouse que os valores do presente estudo foram semelhantes aos descritos por Gottlicher et al. ${ }^{16}$ entre a $17^{\text {a }}$ e $23^{\underline{a}}$ semana, periodo em que os valores relatados situaram-se no intervalo de confiança deste estudo, sendo menores após esse período com diferença de $2 \mathrm{~mm}$ entre os valores médios na $39^{a}$ semana de gestação. Os valores médios do diâmetro biparietal deste estudo equivaleram aos de Grumbach et al. ${ }^{1}$. Em relação aos valores descritos por Reece et al. ${ }^{2}$, o diâmetro biparietal médio deste estudo foi semelhante no período entre a $12^{\text {a }}$ e a $32^{\text {a }}$ semana, sendo menor após o mesmo, ou seja, o limite superior do intervalo de confiança esteve abaixo dos valores descritos pelos autores. A diferença entre os valores médios foi $4 \mathrm{~mm}$ na 39a semana de gestação.

\section{Circunferência cefálica}

Foram obtidos os coeficientes e os respectivos erros-padrão correspondentes ao modelo com melhor ajuste. Esse modelo pode ser escrito como: $\mathrm{CC}$ média $=-6,28864+0,97831 \mathrm{IG}+0,02060 \mathrm{IG}^{2}-$ $0,00053 \mathrm{IG}^{3}$ 
Tabela 1 - Médias e desvios padrão dos parâmetros biométricos observados de 68 fetos gêmeos em cada idade gestacional.

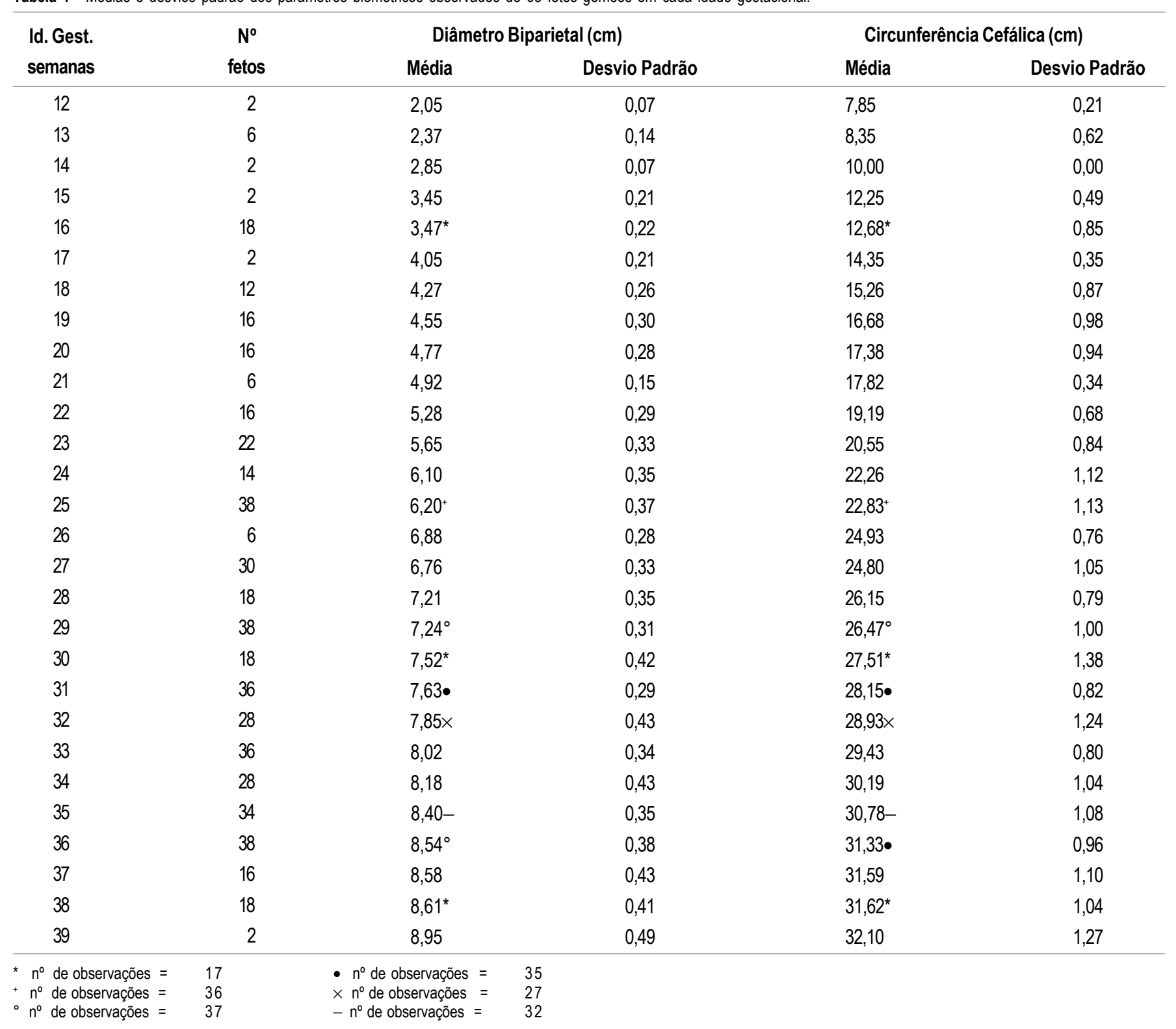

A Tabela 3 apresenta os valores médios estimados e os respectivos intervalos de confiança $(95 \%)$ da circunferência cefálica para idades gestacionais entre 12 e 39 semanas.

A Figura 3 mostra a curva da circunferência cefálica média estimada e os respectivos intervalos de confiança (95\%) entre 12 e 39 semanas de gestação do presente estudo e a de Hadlock et $a 1 .{ }^{6}$ para gestações únicas.

A circunferência cefálica média estimada neste trabalho foi equivalente à sugerida por Hadlock et al. ${ }^{6}$ apenas no periodo entre a $12^{\text {a }} \mathrm{e}$ 25a semanas de gestação, sendo menor após esse período, ou seja, o limite superior do intervalo de confiança do presente estudo esteve abaixo dos valores descritos por Hadlock et al. ${ }^{6}$. Na 39a semana, a diferença entre os valores médios foi $2,0 \mathrm{~cm}$.

A circunferência cefálica média estimada no presente estudo foi comparada aos valores descritos para a gestação gemelar por Rodis et al. ${ }^{17} \mathrm{e}$ Reece et al. ${ }^{2}$ (Figura 4). Os valores médios da circunferência cefálica obtidas nesse trabalho em relação aos de Rodis et al. ${ }^{17}$ foram maiores entre a 15a e $18^{\text {a }}$ semana e semelhantes após esse período. Constatou-se, ainda, que a circunferência cefálica obtida foi semelhante à de Reece et $a .^{2}$ entre 27 e 33 semanas de gestação, sendo maior antes e menor após esse periodo, ocorrendo uma diferença entre os valores médios de $1,9 \mathrm{~cm}$ na 39a semana de gestação. 
Tabela 2 - Valores médios estimados e respectivos limites de confiança (95\%) do diâmetro biparietal de fetos gêmeos entre 12 e 39 semanas.

\begin{tabular}{|c|c|c|c|}
\hline \multirow{2}{*}{$\begin{array}{c}\text { Idade } \\
\text { Gestacional } \\
\text { (semanas) }\end{array}$} & \multirow{2}{*}{$\begin{array}{c}\text { Diâmetro } \\
\text { Biparietal } \\
\text { (cm) }\end{array}$} & \multicolumn{2}{|c|}{$\begin{array}{l}\text { Limites de Confiança } \\
\qquad(95 \%)\end{array}$} \\
\hline & & Inferior & Superior \\
\hline 12 & 2,1 & 1,9 & 2,2 \\
\hline 13 & 2,4 & 2,3 & 2,5 \\
\hline 14 & 2,8 & 2,6 & 2,9 \\
\hline 15 & 3,1 & 3,0 & 3,2 \\
\hline 16 & 3,4 & 3,3 & 3,5 \\
\hline 17 & 3,8 & 3,7 & 3,9 \\
\hline 18 & 4,1 & 4,0 & 4,2 \\
\hline 19 & 4,4 & 4,4 & 4,5 \\
\hline 20 & 4,8 & 4,7 & 4,9 \\
\hline 21 & 5,1 & 5,0 & 5,2 \\
\hline 22 & 5,4 & 5,3 & 5,5 \\
\hline 23 & 5,7 & 5,6 & 5,8 \\
\hline 24 & 6,0 & 5,9 & 6,1 \\
\hline 25 & 6,3 & 6,2 & 6,3 \\
\hline 26 & 6,5 & 6,4 & 6,6 \\
\hline 27 & 6,8 & 6,7 & 6,9 \\
\hline 28 & 7,0 & 6,9 & 7,1 \\
\hline 29 & 7,3 & 7,2 & 7,4 \\
\hline 30 & 7,5 & 7,4 & 7,6 \\
\hline 31 & 7,7 & 7,6 & 7,8 \\
\hline 32 & 7,9 & 7,8 & 8,0 \\
\hline 33 & 8,0 & 7,9 & 8,1 \\
\hline 34 & 8,2 & 8,1 & 8,3 \\
\hline 35 & 8,3 & 8,2 & 8,4 \\
\hline 36 & 8,5 & 8,3 & 8,6 \\
\hline 37 & 8,6 & 8,4 & 8,7 \\
\hline 38 & 8,6 & 8,5 & 8,8 \\
\hline 39 & 8,7 & 8,6 & 8,8 \\
\hline
\end{tabular}

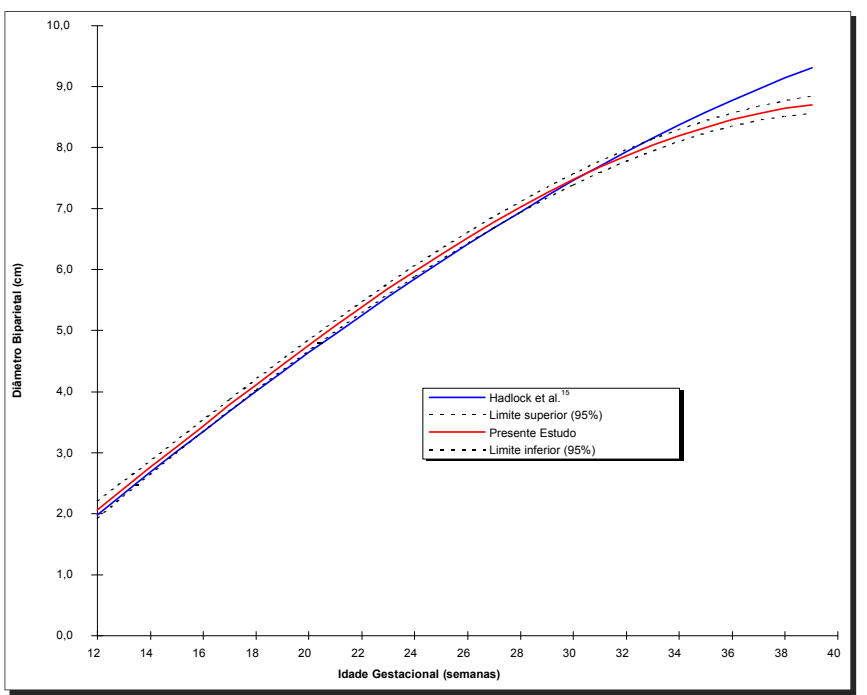

Figura 1 - Curva de crescimento do diâmetro biparietal médio com intervalos de confiança de $95 \%$ do presente estudo e a curva de Hadlock et al. ${ }^{15}$ para fetos únicos.

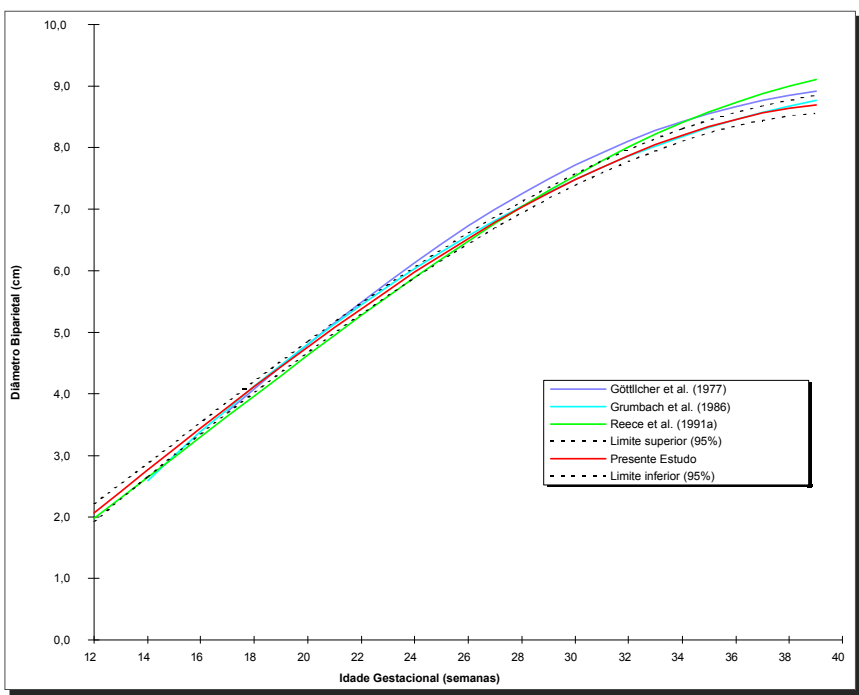

Figura 2 - Curva de crescimento do diâmetro biparietal médio com intervalos de confiança de $95 \%$ do presente estudo e curvas de Gottlicher et al. ${ }^{16}$, Grumbach et al. ${ }^{1}$ e Reece et al. ${ }^{2}$ para fetos gêmeos.

Tabela 3 - Valores médios estimados e respectivos limites de confiança (95\%) da circunferência cefálica de fetos gêmeos entre 12 e 39 semanas.

\begin{tabular}{|c|c|c|c|}
\hline \multirow{2}{*}{$\begin{array}{l}\text { Idade Gestacional } \\
\text { (semanas) }\end{array}$} & \multirow{2}{*}{$\begin{array}{l}\text { Circunferência } \\
\text { Cefálica }(\mathrm{cm})\end{array}$} & \multicolumn{2}{|c|}{ Limites de Confiança (95\%) } \\
\hline & & Inferior & Superior \\
\hline 12 & 7,5 & 7,1 & 8,0 \\
\hline 13 & 8,8 & 8,4 & 9,1 \\
\hline 14 & 10,0 & 9,7 & 10,3 \\
\hline 15 & 11,2 & 10,9 & 11,6 \\
\hline 16 & 12,5 & 12,2 & 12,8 \\
\hline 17 & 13,7 & 13,4 & 14,0 \\
\hline 18 & 14,9 & 14,6 & 15,2 \\
\hline 19 & 16,1 & 15,8 & 16,4 \\
\hline 20 & 17,3 & 17,0 & 17,6 \\
\hline 21 & 18,5 & 18,2 & 18,8 \\
\hline 22 & 19,6 & 19,3 & 19,9 \\
\hline 23 & 20,7 & 20,4 & 21,0 \\
\hline 24 & 21,8 & 21,5 & 22,1 \\
\hline 25 & 22,8 & 22,6 & 23,1 \\
\hline 26 & 23,8 & 23,6 & 24,1 \\
\hline 27 & 24,8 & 24,5 & 25,1 \\
\hline 28 & 25,7 & 25,5 & 26,0 \\
\hline 29 & 26,6 & 26,3 & 26,9 \\
\hline 30 & 27,4 & 27,1 & 27,7 \\
\hline 31 & 28,2 & 27,9 & 28,4 \\
\hline 32 & 28,9 & 28,6 & 29,2 \\
\hline 33 & 29,5 & 29,3 & 29,8 \\
\hline 34 & 30,1 & 29,9 & 30,4 \\
\hline 35 & 30,7 & 30,4 & 30,9 \\
\hline 36 & 31,1 & 30,8 & 31,4 \\
\hline 37 & 31,5 & 31,2 & 31,8 \\
\hline 38 & 31,8 & 31,4 & 32,2 \\
\hline 39 & 32,0 & 31,6 & 32,4 \\
\hline
\end{tabular}




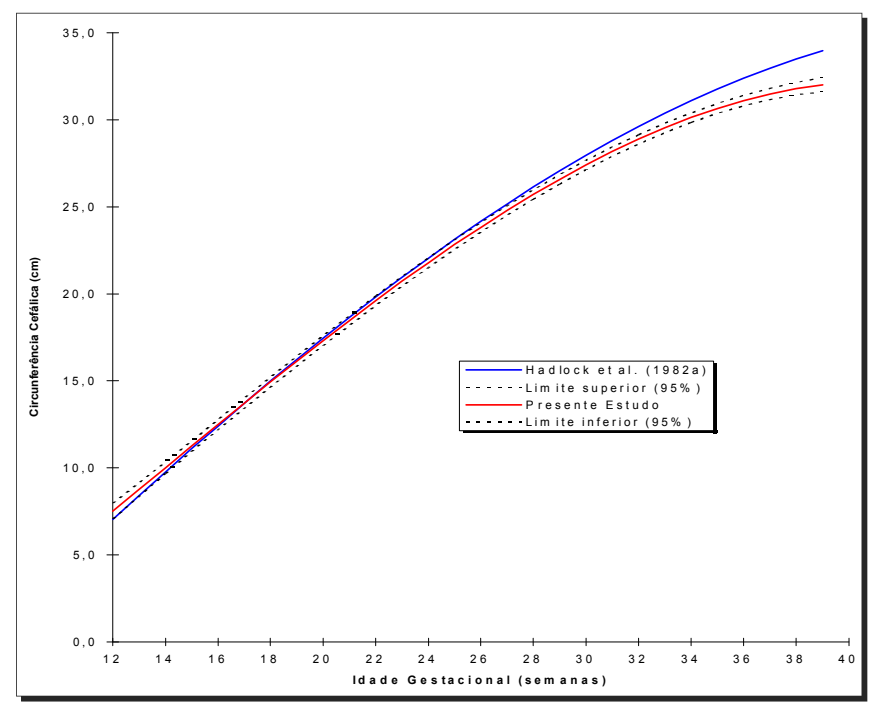

Figura 3 - Curva de crescimento da circunferência cefálica média com intervalos de confiança de $95 \%$ do presente estudo e a curva de Hadlock et al. ${ }^{6}$ para fetos únicos.

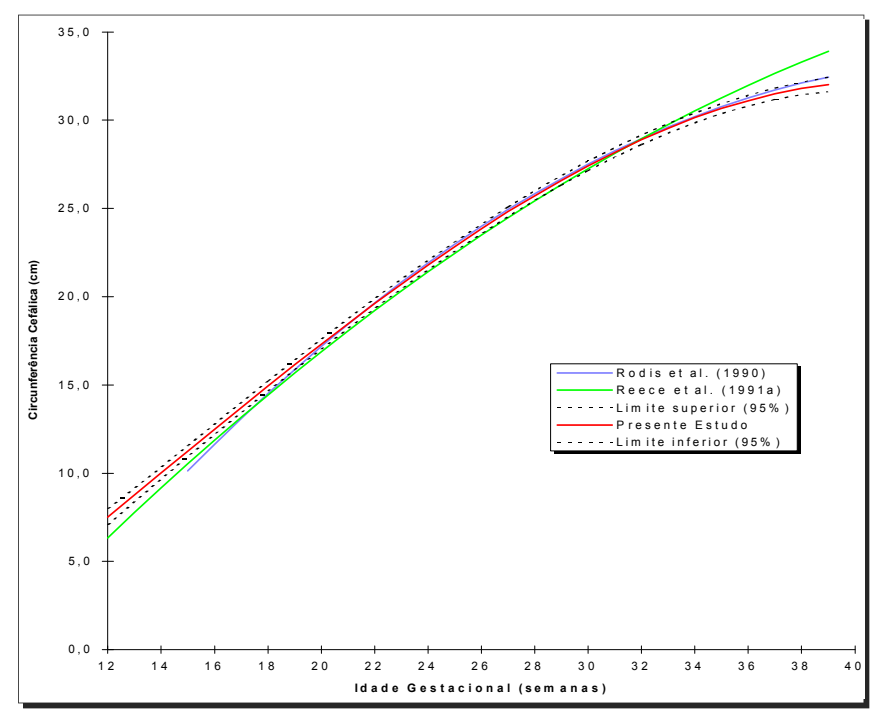

Figura 4 - Curva de crescimento da circunferência cefálica média com intervalos de confiança de $95 \%$ do presente estudo e curva de Rodis et al. ${ }^{17}$ e Reece et al. ${ }^{2}$ para fetos gêmeos.

\section{Discussão}

Analisando-se a literatura pertinente, detecta-se erros cometidos na obtenção de algumas curvas de crescimento dos parâmetros biométricos ultrasonográficos para gestações gemelares. As principais são a omissão de critérios de inclusão e exclusão da população analisada por alguns autores ${ }^{17,18,19,20,21,22,23}$ ou a inclusão de gestações gemelares com patologias fetomaternas ${ }^{24,25,26,27}$, fatos que podem comprometer o resultado dos trabalhos.

Apesar de alguns estudos serem longitudinais, em nenhum deles os exames ultra- sonográficos são realizados por um único operador como nesta pesquisa. Deter et al. ${ }^{28}$ constatam diferenças de até $8 \%$ entre observadores ao passo que Hadlock et al. ${ }^{7}$ descrevem erro de no máximo $0,7 \%$ em exames ultra-sonográficos efetuadas pelo mesmo observador, demonstrando que medidas efetuadas por uma única pessoa tendem a minimizar o erro observacional.

As curvas obtidas a partir da análise longitudinal descrevem o crescimento individual e são mais apropriadas para a construção de curvas do crescimento médio, que representam a população como um todo ${ }^{8,29}$. Em estudos longitudinais, observações repetidas em um mesmo individuo podem propiciar correlações entre as medidas. Neste caso a análise estatística por meio do modelo de efeitos aleatórios é mais adequada do que a regressão linear simples, a qual pressupõe variância constante e observações não-correlacionadas.

A maioria dos estudos constata valores menores do diâmetro biparietal médio nos fetos gêmeos comparados aos únicos durante o terceiro trimestre ${ }^{1,16,18,22,25,26,30,31}$. Outros verificam que $o$ diâmetro biparietal médio de gêmeos é menor durante todo o período gestacional em relação a fetos únicos ${ }^{19,20,32}$, enquanto alguns ${ }^{2,21,23,24}$ não encontram diferenças significativas.

A determinação isolada do diâmetro biparietal médio pode não avaliar adequadamente o crescimento fetal devido à possibilidade da ocorrência de dolicocefalia. A circunferência reflete melhor o volume cefálico e é menos dependente do formato da cabeça do que a medida do diâmetro biparietal ${ }^{33}$. Muitos autores têm demonstrado que a circunferência cefálica é um dos parâmetros individuais mais confiáveis para a estimativa da idade gestacional ${ }^{6,34,35}$. Em relação aos estudos sobre a medida da circunferência cefálica em fetos de gestações gemelares, Rodis et al. ${ }^{17}$ construiram uma curva da circunferência cefálica em relação à idade gestacional. Reece et al. ${ }^{2}$ não encontram diferenças na circunferência cefálica entre gestações gemelares e únicas.

Este trabalho sugeriu que as curvas do crescimento médio do diâmetro biparietal e da circunferência cefálica de Hadlock et al. ${ }^{6,15}$, bastante utilizadas na prática diária, não parecem apropriadas para a avaliação de gestação gemelar da população analisada, pois levaram à subestimação da idade gestacional principalmente no terceiro trimestre da gestação. A presente análise não permitiu conclusões a respeito da comparação de fetos gêmeos e fetos únicos, uma vez que o estudo de Hadlock et al. ${ }^{6,15}$, além de ser transversal, compreende uma população que diverge da presente pesquisa em inúmeros aspectos (étnicos, socioculturais, econômicos etc.). No futuro, a ob- 
tenção da curva do crescimento a partir de parâmetros ultra-sonográficos de fetos únicos da população local, de modo prospectivo e longitudinal, possibilitará comparação adequada do crescimento fetal da gestação gemelar em relação à única.

O fato de alguns dos valores do presente estudo serem menores do que os de outros autores pode decorrer de diferenças étnicas e prováveis desvantagens socioeconômicas da população estudada já que os trabalhos disponíveis para comparação foram realizados em países desenvolvidos. Porém pequenas diferenças entre as curvas talvez não fossem significativas se se pudesse dispor dos intervalos de confiança das curvas médias estimadas descritas pelos autores. Foi interessante observar-se que os valores do diâmetro biparietal médio obtidos por Grumbach et al. ${ }^{1} \mathrm{em}$ 103 gestações gemelares e da circunferência cefálica média obtidos por Rodis et al. ${ }^{17}$ em 60 gestações gemelares de população norte-americana estivessem tão próximos aos valores médios do presente estudo.

\section{SUMMARY}

Purpose: to estimate growth curves and tables of average biparietal diameter and average head circumference of twin gestations and to compare them with published growth curves for singletons and twins.

Methods: growth curves and tables of average biparietal diameter and average head circumference were obtained from sonographic examinations of 34 patients with twin gestations without maternal and fetal complications. Sonographic examinations were performed each 2 or 3 weeks by a single observer. The parameters were compared with existing growth curves for singletons and twins.

Results: curves and tables of biparietal diameter and head circumference in relation to gestational age were obtained. The study revealed difference in growth pattern mainly regarding the $3^{\text {rd }}$ trimester in relation to singletons (Hadlock et al. ${ }^{15}$ ). The difference between the average values were 6 $\mathrm{mm}$ (biparietal diameter) and $2.0 \mathrm{~cm}$ (head circumference) at the $39^{\text {th }}$ week of gestation. Also, some differences were observed relative to the twin studies.

Conclusion: this study revealed that growth curves for average biparietal diameter and average head circumference of singletons are not appropriate to determine gestational age in twin gestations of the studiced population principally at the $3^{\text {rd }}$ trimester.

KEY WORDS: Twin pregnancy. Ultrasonography. Fetal growth. Biparietal diameter.

\section{Referências}

1. Grumbach K, Coleman BG, Arger PH, Mintz MC, Gabbe SV, Mennuti MT. Twin and singleton growth patterns compared using US. Radiology 1986; 158:237-41.

2. Reece EA, Yarkoni S, Abdalla M, Gabrielli S, Holford T, O'Connor TZ, et al. A prospective longitudinal study of growth in twin gestations compared with growth in singleton pregnancies I.The fetal head. J Ultrasound Med 1991; 10:439-43.

3. Hadlock FP, Deter RL, Harrist RB, Park SK. Estimating fetal age: computer-assisted analysis of multiple fetal growth parameters. Radiology 1984; 152:497-501.

4. Robinson HP, Fleming JE. A critical evaluation of sonar crown-rump length measurements. $\mathrm{Br} \mathrm{J}$ Obstet Gynaecol 1975; 82:702-10.

5. Kurtz AB, Wapner RJ, Kurtz RJ, Dershaw DD, Rubin CS, Cole-Beuglet C, et al. Analysis of biparietal diameter as an accurate indicator of gestational age. J Clin Ultrasound 1980; 8:319-26.

6. Hadlock FP, Deter RL, Harrist RB, Park SK. Fetal head circumference: relation to menstrual age. AJR (Am J Roentgenol) 1982; 138:649-53.

7. Hadlock FP, Deter RL, Harrist RB, Park SK. Fetal abdominal circumference as a predictor of menstrual age. AJR (Am J Roentgenol) 1982; 139:367-70.

8. Hadlock FP, Harrist RB, Deter RL, Park SK. Fetal femur length as a predictor of menstrual age: sonographically measured. ARJ (Am J Roentgenol) 1982; 138:875-8.

9. WHO: recommended definitons, terminology and format for statistical tables related to the perinatal and use of a new certificate for cause of perinatal deaths. Acta Obstet Gynecol Scand $1977 ; 56: 247-53$.

10.Kurtz AB, Needleman L. Avaliação da idade fetal por ultra-som. In: Callen PW, editor. UltraSonografia em Obstetrícia e Ginecologia. São Paulo: Santos, 1991. p.47-64.

11.Hadlock FP, Kent WR, Loyd JL, Harrist RB, Deter RL, Park SK. An evaluation of two methods for measuring fetal head and body circumferences. J Ultrasound Med 1982; 1:359-60.

12. Capurro H, Konichezky S, Fonseca D, CaldeyroBarcia R. A simplified method for diagnosis of gestational age in the newborn infant. J Pediatr 1978; 93:120-2.

13.Singer JM, Andrade DA. Analysis of longitudinal data. In: Rao CR, Sen PK, ed. Bio-enviromental and public health statistics. Amsterdam, North Holland, 1999. (Handbook of statistics, v.17) [In press] 
14.Diggle PJ, Liang KY, Zeger SL. Analysis of longitudinal data. Oxford: Oxford University Press; 1994.

15.Hadlock FP, Deter RL, Harrist RB, Park SK. Fetal biparietal diameter: a critical re-evaluation of the relation to menstrual age by means of real-time ultrasound. J Ultrasound Med 1982; 1:97-104.

16.Gottlicher S, Madjaric J, Krone HA. Der biparietale Durchmesser des fetalen Kopfes bei Zwillingen und Einlingen im Verlauf der Schwangerschaft. Eine vergleichende Studie. Geburtshilfe Frauenheilkd 1977; 37:762-7.

17.Rodis JF, Vintzileos AM, Campbell WA, Pinette MG, Nochimson DJ. Intrauterine fetal growth in concordant twin gestations. Am J Obstet Gynecol 1990; 162:1025-9.

18.Bleker OP, Kloosternan GJ, Huidekoper BL, Breur W. Intrauterine growth of twins as estimated from birthweigth and the fetal biparietal diameter. Eur J Obstet Gynecol Reprod Biol 1977; 7:85-90.

19.Schneider L, Bessis R, Tabaste JL, Sarramont MF, Papiernik E, Baudet J, et al. Echographic survey of twin fetal growth: a plea for specific charts for twins. In: Nance WE, editor. Twin Research: Clinical Studies. New York: Alan R. Liss; 1978. p.137-41.

20.Divers WA, Hemsell DL. The use of ultrasound in multiple gestations. Obstet Gynecol 1979; 53:500-4.

21.Crane JP, Tomich PG, Kopta M. Ultrasonic growth patterns in normal and discordant twins. Obstet Gynecol 1980; 55:678-83.

22. Secher NJ, Kaern J, Hansen PK. Intrauterine growth in twin pregnancies: prediction of fetal growth retardation. Obstet Gynecol 1985; 66:63-8.

23. Shah YG, Graham D, Stinson SK, Render TD. Biparietal diameter growth in uncomplicated twin gestation. Am J Perinatol 1987; 4:229-32.

24.Scheer K. Ultrasound in twin gestation. J Clin Ultrasound 1974; 2:197-9.
25.Grennert L, Persson P, Gennser G. Intrauterine growth of twins judged by BPD measurements. Acta Obstet Gynecol Scand 1978; 78(Suppl):28-32.

26.Houlton MCC, Marivate M, Philpott RH. The prediction of fetal growth retardation in twin pregnancy. Br J Obstet Gynaecol 1981; 88:264-73.

27.Haines CJ, Langlois SL, Jones WR. Ultrasonic measurement of fetal femoral length in singleton and twin pregnancies. Am J Obstet Gynecol 1986; 155:838-41.

28.Deter RL, Harrist RB, Hadlock FP, Carpenter RJ. Fetal head and abdominal circumferences: I. Evaluation of measurement errors. J Clin Ultrasound 1982; 10:357-63.

29.Deter RL, Harrist RB, Hadlock FP, Poindexter AN. Longitudinal studies of fetal growth with the use of dynamic image ultrasonography. Am J Obstet Gynecol 1982; 143:545-54.

30.Socol ML, Tamura RK, Sabbagha RE, Chen T, Vaisrub N. Diminished biparietal diameter and abdominal circumference growth in twins. Obstet Gynecol 1984; 64:235-8.

31.Mordel N, Laufer N, Zajicek G, Shalev Z, Lewin A, Schenker JG, et al. Sonographic growth curves of triplet conceptions. Am J Perinatol 1993; 10:239-42.

32.Leveno KJ, Santos-Ramos R, Duenhoelter JH, Reisch JS, Whalley PJ. Sonar cephalometry in twins: a table of biparietal diameters for normal twin fetuses and a comparison with singletons. Am J Obstet Gynecol 1979; 135:727-30.

33. Hadlock FP, Deter RL, Carpenter RJ, Park SK. Estimating fetal age: effect of head shape on BPD. ARJ Am J Roentgenol 1981; 137:83-5.

34.Law RG, MacRae KD. Head circumference as an index of fetal age. J Ultrasound Med 1982; 1:281-8.

35.Hill LM, Guzick D, Hixson J, Peterson CS, Rivello DM. Composite assessment of gestational age: a comparison of institutionally derived and published regression equations. Am J Obstet Gynecol 1992; 166:551-5. 